Pacific Journal of Mathematics

GROUPS WITH FINITELY MANY AUTOMORPHISMS 


\section{GROUPS WITH FINITELY MANY AUTOMORPHISMS}

\section{J. L. ALPERIN}

1. Introduction. The connection between the structure of a group and the structure of its endomorphisms or group of automorphisms is a very interesting question but one to which there is as yet a scarcity of answers. We present here two theorems pertaining to this problem. We first show that a finitely generated group has a finite automorphism group if and only if it is a finite and central extension of a cyclic group. The restriction to finitely generated groups is essential. Indeed, there exist indecomposable torsion-free abelian groups of rank the cardinal of the continuum whose automorphism groups are cyclic of order two (see [2], p. 180, 18(b)). The second result which we present is a new and much simpler proof of a theorem to be found in a paper of Baer [1]; namely, a group possessing only finitely many endomorphisms is itself finite.

Before discussing these theorems we shall describe the notation to be used in this paper. Throughout, $G$ will denote a group with center $Z$. Let coset representatives $g_{\alpha}, g_{\beta}, \cdots$ of $Z$ in $G$ be chosen for all $\alpha, \beta, \cdots$ elements of $G / Z$ such that $g_{1}=1$. Let $M=\left\{m_{\alpha, \beta}\right\}$ be the corresponding factor set. For the theory of such factor sets, essential to the following, the reader should consult Kurosh [4] or M. Hall [3]. Finally, for any group $H$, let $\operatorname{Aut}(H)$ be the automorphism group of $H$.

2. Preliminary lemmas. The first lemma follows immediately from the definition of equivalence of factor sets.

Lemma 1. Let $N$ and $R$ be factor sets from the group $B$ into the abelian group $A_{1} \times A_{2}$. Let $N_{1}, N_{2}, R_{1}, R_{2}$ be the factor sets from $B$ into $A_{1}$ and $A_{2}$ obtained by taking components of $N$ and $R$. Then $N$ and $R$ will be equivalent if and only if $N_{1}$ is equivalent with $R_{1}$ and $N_{2}$ with $R_{2}$.

The remaining lemmas are, I believe, entirely or in part scattered throughout the literature. We include proofs for the convenience of the reader.

LEMMA 2. Let $\psi$ be an endomorphism of $Z$ such that the factor sets $M$ and $\psi(M)=\left\{\psi\left(m_{\alpha, \beta}\right)\right\}$ of $G$ are equivalent. Then $\psi$ may be extended to an endomorphism of $G$ and if $\psi$ is an automorphism of $Z$ then it may be extended to an automorphism of $G$.

Received February 17, 1961. National Science Foundation Predoctoral Fellow. 
Proof. First note that it is clear from the definition of factor set that $\psi(M)$ is a factor set. Since $M$ and $\psi(M)$ are assumed to be equivalent we may choose for each $\alpha \in G / Z$ an element $c_{\alpha} \in Z$ such that

$$
\psi\left(m_{\alpha, \beta}\right)=c_{\alpha \beta}^{-1} m_{\alpha, \beta} c_{\alpha} c_{\beta}
$$

for all $\alpha, \beta \in G \mid Z$. Now every element $g \in G$ can be uniquely expressed in the form $g=g_{\alpha}$ a where $\alpha \in G \mid Z$ and $a \in Z$. Thus we may define a mapping $\varphi$ of $G$ into itself according to the rule, for all $g \in G$,

$$
\varphi(g)=g_{\alpha} c_{\alpha} \psi(a) \text {. }
$$

Then $\varphi$ is an endomorphism of $G$ since if $g=g_{\alpha} a, h=g_{\beta} b$ are elements of $G$, where $\alpha, \beta \in G \mid Z, a, b \in Z$ then

$$
\begin{aligned}
\varphi(g h) & =\varphi\left(g_{\alpha} a g_{\beta} b\right) \\
& =\varphi\left(g_{\alpha \beta} m_{\alpha, \beta} a b\right) \\
& =g_{\alpha \beta} c_{\alpha \beta} \psi\left(m_{\alpha, \beta} a b\right) \\
& =g_{\alpha \beta} m_{\alpha, \beta} c_{\alpha} c_{\beta} \psi(a) \psi(b) \\
& =g_{\alpha} c_{\alpha} \psi(a) g_{\beta} c_{\beta} \psi(b) \\
& =\varphi(g) \varphi(h) .
\end{aligned}
$$

Since $g_{1}=1$ implies $m_{1,1}=g_{1}^{-1} g_{1} g_{1}=1$ and so $c_{1}=1$, we have that $\varphi$ extends $\psi$ to $G$.

Finally, suppose $\psi$ is an automorphism of $G$. Let $g=g_{\alpha} a$ be an arbitrary element of $G$, as above. Then $\varphi(g)=1$ implies that $g_{\alpha} c_{\alpha} \psi(\alpha)=1$ and hence $\alpha=1$ because $\alpha$ is the image of $g_{\alpha} c_{\alpha} \psi(a)$ in $G / Z$. Therefore $g_{1}=c_{1}=1$ so we have $\psi(a)=1$ and $g=g_{\alpha} a=1$. $\varphi$ is consequently a one-to-one mapping of $G$ into itself. To conclude we prove that $g$ is the image of some element under $\varphi$. Choose $b \in Z$ such that $\psi(b)=$ $c_{\alpha}^{-1} a$. Then $\varphi\left(g_{\alpha} b\right)=g_{\alpha} c_{\alpha} \psi(b)=g_{\alpha} a=g$.

Lemma 3. Let $A$ be a central and characteristic subgroup of the group $H$. The group $K$ of those automorphisms of $H$ which leave $A$ element-wise fixed and which induce the identity automorphism on $H \mid A$ is naturally isomorphic to the group $\operatorname{Hom}(H \mid A, A)$ of homomorphisms of $H \mid A$ into $A$.

Proof. If $\sigma \in \operatorname{Hom}(H / A, A)$ define a mapping $\theta$ of $H$ into itself by $\theta(h)=h \sigma(h A)$ for $h \in H$. $\quad \theta$ will be an element of $K$, as can be verified directly, and the correspondence of $\sigma$ with $\theta$ is the required isomorphism.

Corollary. If $\operatorname{Aut}(A), \operatorname{Aut}(H \mid A)$, and $\operatorname{Hom}(H \mid A, A)$ are finite then $\operatorname{Aut}(H)$ is finite. 
Proof. If $\theta \in \operatorname{Aut}(H)$ let $\theta_{1}$ be the restriction of $\theta$ to $A$ and $\theta_{2}$ the automorphism of $H \mid A$ induced by $\theta$. Then the mapping sending $\theta$ to $\left(\theta_{1}, \theta_{2}\right) \in \operatorname{Aut}(A) \times \operatorname{Aut}(H \mid A)$ is a homomorphism with kernel $K$. Hence, the product of the orders of $\operatorname{Aut}(A), \operatorname{Aut}(H \mid A)$, and $\operatorname{Hom}(H \mid A, A)$ is a bound for the order of $\operatorname{Aut}(H)$.

\section{Finite automorphism groups.}

THEOREM 1. A finitely generated group G has a finite automorphism group if and only if it has a central cyclic subgroup of finite index.

Proof. First we shall demonstrate the sufficiency of these conditions. In so doing, we may assume $G$ is infinite and therefore a finite and central extension of an infinite cyclic group. The center $Z$ of $G$ is of finite index in $G$ so by Schreier's subgroup theorem (3; p. 97) is finitely generated. Hence $Z$ is the direct sum of a finite abelian group $T$ and an infinite cyclic group. Consequently, $\operatorname{Aut}(G / Z)$ and $\operatorname{Hom}(G \mid Z, Z)=$ $\operatorname{Hom}(G \mid Z, T)$ are finite. To conclude, we can apply the above Corollary once we prove that $\operatorname{Aut}(Z)$ if finite. However, this follows from another application of the Corollary with $A=T$ and $H=Z$.

The necessity of the condition is more difficult to prove. If $\operatorname{Aut}(G)$ is finite then $G / Z$ is finite being isomorphic to the group of inner automorphisms of $G$. Again, by Schreier's theorem, $Z$ will be finitely generated. We may assume, in contradiction to the theorem, that $Z$ in a direct decomposition into cyclic groups, contains two or more infinite cyclic factors. In fact let $Z=W \times(a) \times(b)$ where $(a)$ and $(b)$ are the subgroups generated by elements $a$ and $b$ of infinite order and $W$ is a subgroup of $Z$. We need only show that infinitely many automorphisms of $Z$, which are the identity on $W$ and map the group $(a, b)$ generated by $a$ and $b$ onto itself, may be extended to automorphisms of $G$.

For the remainder of this proof we shall use the additive notation for $Z$. Write the factor set $M$ as $m_{\alpha, \beta}=w_{\alpha, \beta}+r_{\alpha, \beta} a+t_{\alpha, \beta} b$ where $w_{\alpha, \beta} \in W, s_{\alpha, \beta}$ and $t_{\alpha, \beta}$ are integers. Let the automorphism $\theta$ of $Z$ be defined by $\theta(w)=w$ for $w W, \theta(a)=m a+n b, \theta(b)=p a+q b$ where $m, n, p$, and $q$ are integers such that $|m q-n p|=1$. The factor set $\theta(M)$ is then expressible as $\theta\left(m_{\alpha, \beta}\right)=w_{\alpha, \beta}+s_{\alpha, \beta}^{\prime} a+t_{\alpha, \beta}^{\prime} b$ where $s_{\alpha, \beta}^{\prime}=m s_{\alpha, \beta}+p t_{\alpha, \beta}$ and $t_{\alpha, \beta}^{\prime}=n s_{\alpha, \beta}+q t_{\alpha, \beta}$. Therefore, $S_{0}=\left\{s_{\alpha, \beta}\right\}, T_{0}=\left\{t_{\alpha, \beta}\right\}$, $S_{0}^{\prime}=\left\{s_{\alpha, \beta}^{\prime}\right\}$, and $T_{0}^{\prime}=\left\{t_{\alpha, \beta}^{\prime}\right\}$ are factor sets of $G / Z$ with integral values and $S_{0}^{\prime}=m S_{0}+p T_{0}, T_{0}^{\prime}=n S_{0}+q T_{0}$. By Lemmas 1 and 2 the proof is now reduced to the following problem: Find infinitely many quadruplets $(m, n, p, q)$ of integers such that $|m q-n p|=1$ and the factor sets $m S_{0}+p T_{0}$ and $n S_{0}+q T_{0}$ are equivalent with the factor sets $S_{0}$ and $T_{0}$ respectively. That is, the factor sets $(m-1) S_{0}+p T_{0}$ and $n S_{0}+(q-1) T_{0}$ are both equivalent to the trivial factor sets. However, if $G / Z$ has order 
$h$ then $h S_{0}$ and $h T_{0}$ both are equivalent to the trivial factor set $(3 ; \mathrm{p}$. 223). Thus, for any integer $\mathrm{k}$ the following values for $m, n, p$ and $q$ suffice:

$$
\begin{aligned}
& m=-h^{2}-h k+1 \\
& n=h \\
& p=-h^{2} k-h k^{2}-h \\
& q=h k+1,
\end{aligned}
$$

and the theorem is proved.

\section{Groups with finitely many endomorphisms.}

THEOREM 2. A group G which has only finitely many endomorphisms is itself finite.

Proof. The group $G$ will then have only finitely many inner automorphisms so $Z / G$ will be finite, say of order $n$. We shall once again use multiplicative notation for $Z$. For any positive integer $k$ the factor sets $M$ and $M^{k n+1}$ are equivalent (as above) so that we may, by Lemma 2 , extend to endomorphisms of $G$ the endomorphisms $\theta(k)$ of $Z$ which map $z$ to $z^{k n+1}$ for $z \in Z$. If $Z$ contains at least one element of infinite order then all the $\theta(k)$ will be distinct. Therefore, we shall assume that $Z$ is a torsion group.

Suppose that we can decompose $Z$ as the direct product $\Pi_{\gamma} A_{\gamma}$ of infinitely many nontrivial factors. The elements $m_{\alpha, \beta}$ of the factor set $M$ will have components in only finitely many of the factors $A_{\gamma}$, because there are only finitely many elements $m_{\alpha, \beta}$. If $A_{\delta}$ is any factor containing no component of any element $m_{\alpha, \beta}$ then we can extend the endomorphism $\psi$ of $Z$ to $G$ where $\psi$ is defined by

$$
\begin{aligned}
& \psi(a)=a \text { if } a \in A_{\rho}, \quad \rho \neq \delta \\
& \psi(a)=1 \text { if } a \in A_{\delta} .
\end{aligned}
$$

This will give us infinitely many endomorphisms of $G$.

If $Z$ is of hounded order then ${ }^{1}$ it is the direct sum of cyclic groups: so that either $Z$ is finite or the preceding paragraph applies. If $Z$ is of unbounded order write $Z$ as the direct product of its Sylow subgroups. In this case either infinitely many of these subgroups are nontrivial and the above remarks pertain or for some prime $p$ the $p$-Sylow subgroup is of unbounded order. Choose then elements $x_{1}, x_{2}, \cdots$ of $Z$ such that $x_{i}$ has order $p^{i}$. The endomorphisms $\theta\left(p^{j}\right)$ defined in the first paragraph will all be distinct. For if $n=p^{r} n_{1}$ where $p$ and $n_{1}$ are coprime then

\footnotetext{
1 For basic results on abelian groups see [2].
} 
$\theta\left(p^{j}\right)\left(x_{i}\right)=x_{i}$ if and only if $i \leqq j+r$. These endomorphisms $\theta\left(p^{j}\right)$ may, as we said, be extended to $G$ so $G$ again has infinitely many endomorphisms. Thus $Z$ is finite so $G$ is also finite.

In closing, the author should like to express his appreciation for the advice and criticism of Dr. G. Baumslag.

\section{REFERENCES}

1. R. Baer, Finite extensions of Abelian groups with minimum condition, Trans. Amer. Math. Soc., 79 (1955), 521-40.

2. L. Fuchs, Abelian Groups, Budapest, 1958.

3. M. Hall, The Theory of Finite Groups. Macmillan, New York, 1959.

4. A. G. Kurosh, The Theory of Groups, Chelsea, New York, 1956.

PRINCETON UNIVERSITY

UNIVERSITY OF CHICAGO 



\section{PACIFIC JOURNAL OF MATHEMATICS}

\section{EDITORS}

Ralph S. Phillips

Stanford University

Stanford, California

M. G. Arsove

University of Washington

Seattle 5, Washington
A. L. Whiteman

University of Southern Californla

Los Angeles 7, California

Lowell J. Paige

University of California

Los Angeles 24, California

\section{ASSOCIATE EDITORS}

E. F. BECKENBACH

D. DERRY

H. L. ROYDEN

E. G. STRAUS

T. M. CHERRY

M. OHTSUKA

E. SPANIER

F. WOLF

\section{SUPPORTING INSTITUTIONS}

UNIVERSITY OF BRITISH COLUMBIA

STANFORD UNIVERSITY

CALIFORNIA INSTITUTE OF TECHNOLOGY

UNIVERSITY OF CALIFORNIA

MONTANA STATE UNIVERSITY

UNIVERSITY OF TOKYO

UNIVERSITY OF UTAH

UNIVERSITY OF NEVADA

NEW MEXICO STATE UNIVERSITY

OREGON STATE UNIVERSITY

UNIVERSITY OF OREGON

OSAKA UNIVERSITY

WASHINGTON STATE UNIVERSITY

UNIVERSITY OF WASHINGTON

UNIVERSITY OF SOUTHERN CALIFORNIA

AMERICAN MATHEMATICAL SOCIETY CALIFORNIA RESEARCH CORPORATION SPACE TECHNOLOGY LABORATORIES NAVAL ORDNANCE TEST STATION 


\section{Pacific Journal of Mathematics}

\section{Vol. 12, No. $1 \quad$ January, 1962}

Jonathan L. Alperin, Groups with finitely many automorphisms $\ldots \ldots \ldots \ldots \ldots \ldots \ldots \ldots$

Martin Arthur Arkowitz, The generalized Whitehead product ................ 7

John D. Baum, Instability and asymptoticity in toplogical dynamics . . . . . . . . . . 25

William Aaron Beyer, Hausdorff dimension of level sets of some Rademacher series .... $\quad 35$

Frank Herbert Brownell, III, A note on Cook's wave-matrix theorem . . . . . . . . . . . . . 47

Gulbank D. Chakerian, An inequality for closed space curves ................. 53

Inge Futtrup Christensen, Some further extensions of a theorem of Marcinkiewicz ....... 59

Charles Vernon Coffman, Linear differential equations on cones in Banach spaces . . . . . 69

Eckford Cohen, Arithmetical notes. III. Certain equally distributed sets of integers . . . . . 77

John Irving Derr and Angus E. Taylor, Operators of meromorphic type with multiple poles

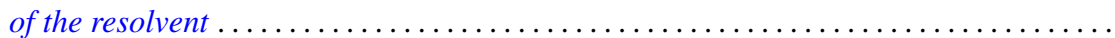

Jacob Feldman, On measurability of stochastic processes in products space .............

Robert S. Freeman, Closed extensions of the Laplace operator determined by a general class of boundary conditions, for unbounded regions ......................

Robert E. Fullerton, Geometric structure of absolute basis systems in a linear topological

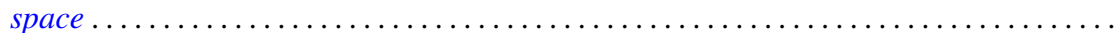

Dieter Gaier, On conformal mapping of nearly circular regions

Andrew Mattei Gleason and Hassler Whitney, The extension of linear functionals defined

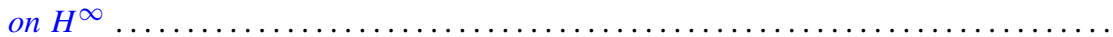

Seymour Goldberg, Closed linear operators and associated continuous linear

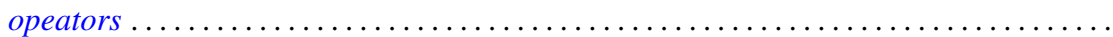

Basil Gordon, Aviezri Siegmund Fraenkel and Ernst Gabor Straus, On the determination of sets by the sets of sums of a certain order

Branko Grünbaum, The dimension of intersections of convex sets. .

Paul Daniel Hill, On the number of pure subgroups

Robert Peter Holten, Generalized Goursat problem . .

Alfred Horn, Eigenvalues of sums of Hermitian matrices ...........

Henry C. Howard, Oscillation and nonoscillation criteria for

$$
y^{\prime \prime}(x)+f(y(x)) p(x)=0
$$

Taqdir Husain, $S$-spaces and the open mapping theorem ...

Richard Eugene Isaac, Markov processes and unique stationary probability measures ...

John Rolfe Isbell, Supercomplete spaces ....................

John Rolfe Isbell, On finite-dimensional uniform spaces. II .........

N. Jacobson, A note on automorphisms of Lie algebras ..............

Antoni A. Kosinski, A theorem on families of acyclic sets and its applications

Marvin David Marcus and H. Minc, The invariance of symmetric functions of singular values...

Ralph David McWilliams, A note on weak sequential convergence.

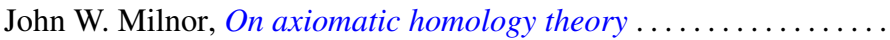

Victor Julius Mizel and Malempati Madhusudana Rao, Nonsymmetric projections in

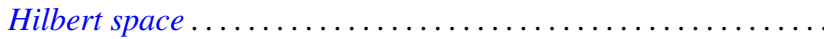

Calvin Cooper Moore, On the Frobenius reciprocity theorem for locally compact

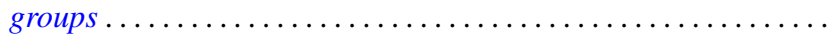

Donald J. Newman, The Gibbs phenomenon for Hausdorff means . 